\title{
Associating Sites of Methanol Maser Emissions at 6.7 GHz
}

\author{
K. Sugiyama ${ }^{* a}$, K. Fujisawa ${ }^{a}$, A. Doi ${ }^{b}$, M. Honma $^{c}$, Y. Isono ${ }^{a}$, N. Mochizuki ${ }^{b}$, \\ Y. Murata ${ }^{b}$, S. Sawada-Satoh ${ }^{a}$, and K. Wajima ${ }^{a}$ \\ ${ }^{a}$ Yamaguchi University, Yamaguchi 753-8512, Japan \\ ${ }^{b}$ The Institute of Space and Astronautical Science, Japan Aerospace Exploration Agency, \\ Kanagawa 229-8510, Japan \\ ${ }^{c}$ National Astronomical Observatory of Japan, Tokyo 181-8588, Japan \\ E-mail: m005wa@yamaguchi-u.ac.jp
}

\begin{abstract}
We have carried out VLBI observations of some methanol maser sources at $6.7 \mathrm{GHz}$ with the Japanese VLBI Network (JVN) using phase-referencing techniques. We superposed the methanol masers on water, hydroxyl masers, radio continuum, and various molecular line emissions with an accuracy of a milliarcsecond (mas) scale, which is enough for investigating a positional relation with a central or exciting star. In this paper, we report results of the superposition in massive star-forming regions (SFRs) Cepheus A (Cep A) and Onsala 1 (ON 1). The methanol maser spots of Cep A showed an arched structure with a scale of 1400 AU. This structure was roughly perpendicular to a radio continuum jet, having an exciting star near the center of the arch, and was coincident with the $\mathrm{CH}_{3} \mathrm{CN}, \mathrm{NH}_{3}$ disks. It is easy to explain that the Cep A methanol masers are associated with a circumstellar disk around a massive young stellar object (YSO). The methanol maser spots of ON 1 formed two main clusters isolated 1800 AU from each other, which corresponded to a red- and a blue-shifted spectral feature, respectively. The direction of the elongation of the isolated clusters coincided with the $\mathrm{H}^{13} \mathrm{CO}^{+}$, SiO molecular outflows, although each spatial size is quite different. Also, the position-velocity diagram along the direction of the molecular outflows roughly coincided. The ON 1 methanol masers may trace an outflow ejected from a massive YSO, while it is also thought to be possible that the masers of this source may trace an expansion of an Ultra-compact (UC) HII region by superposing on the hydroxyl masers.
\end{abstract}

The 9th European VLBI Network Symposium on The role of VLBI in the Golden Age for Radio Astronomy and EVN Users Meeting

September 23-26, 2008

Bologna, Italy

\footnotetext{
* Speaker.
} 


\section{Introduction}

The methanol masers at $6.7 \mathrm{GHz}$ were detected only in massive SFRs and these were thought to trace pre- or early UC HII region phase. This maser, therefore, could be a useful tool for investigations of star-formation around massive YSOs. The site of this maser, however, is still unclear and depends on whether this maser is associated with a circumstellar disk around a massive YSO or shock regions which are formed by outflows ejected from the YSO.

We have carried out VLBI observations of some methanol maser sources at $6.7 \mathrm{GHz}$ with the Japanese VLBI Network (JVN) using phase-referencing techniques. We report results of superposition on water, hydroxyl masers, radio continuum, and various molecular line emissions with an accuracy of a milliarcsecond (mas) scale in the massive SFRs Cep A and ON 1. The VLBI map of methanol masers for Cep A was already obtained with the JVN using phase-referencing techniques [10]. A VLBI observation of methanol masers at $6.7 \mathrm{GHz}$ for ON 1 was carried out on 11 May 2008 using five JVN stations (Yamaguchi, VERA-Mizusawa, -Ogasawara, -Iriki, and -Ishigaki).

\section{Results and Discussions}

The spatial distribution of the Cep A methanol maser spots showed an arched structure with a scale of $1400 \mathrm{AU}$, which was consistent with the result of [13]. The result of superposition on various emissions is shown in Fig. 1(a). The peak of $43 \mathrm{GHz}$ continuum emission, which may be an exciting star [2], was located near center of the arched structure. The elongation of the arched structure was nearly perpendicular to the radio jet (e.g., [2], [11]) at difference of position angle $\sim 70$ degree. The overall distribution of the maser spots coincided with the $\mathrm{CH}_{3} \mathrm{CN}$ and $\mathrm{NH}_{3}$ disks ([8] and [12]) and the velocity range of the spots is similar to that of these disks. The methanol distribution also roughly coincided with the water maser and $\mathrm{SO}_{2}$ disks ([11] and [5]), although the sizes of these disks are about 2 times smaller. It is easy to explain from these results that the Cep A methanol masers are associated with a circumstellar disk. However, a simple velocity gradient like Keplerian rotation was not detected in the methanol maser spots. The disk may rotate with infall or expansion.

The methanol maser spots of ON 1 formed two main clusters isolated 1800 AU from each other, which corresponded to a red- $\left(15 \mathrm{~km} \mathrm{~s}^{-1}\right)$ and a blue-shifted $\left(0 \mathrm{~km} \mathrm{~s}^{-1}\right)$ spectral feature, respectively. The result of superposition on various emissions based on the absolute coordinates obtained by [4 is shown in Fig. 1(b). First, exciting stars are probably different between water masers [7] and methanol masers, and the methanol masers were associated with the UC HII region [1]. The direction of the elongation of the isolated clusters was parallel to the $\mathrm{H}^{13} \mathrm{CO}^{+}, \mathrm{SiO}$ molecular outflows, although each spatial size is quite different. Also, the position-velocity diagram along the direction of the molecular outflows roughly coincided. The ON 1 methanol masers may trace a molecular outflow. On the other hand, the methanol masers coincided with the hydroxyl masers which traced an expansion of the UC HII region by measuring internal proper motions [3]. The ON 1 methanol masers may also trace an expansion of the UC HII region.

We have conducted multi-epoch VLBI observations of $6.7 \mathrm{GHz}$ methanol masers with the JVN in order to measure internal proper motions and will report the results for discussing the associated sites of this maser in a forthcoming paper. 

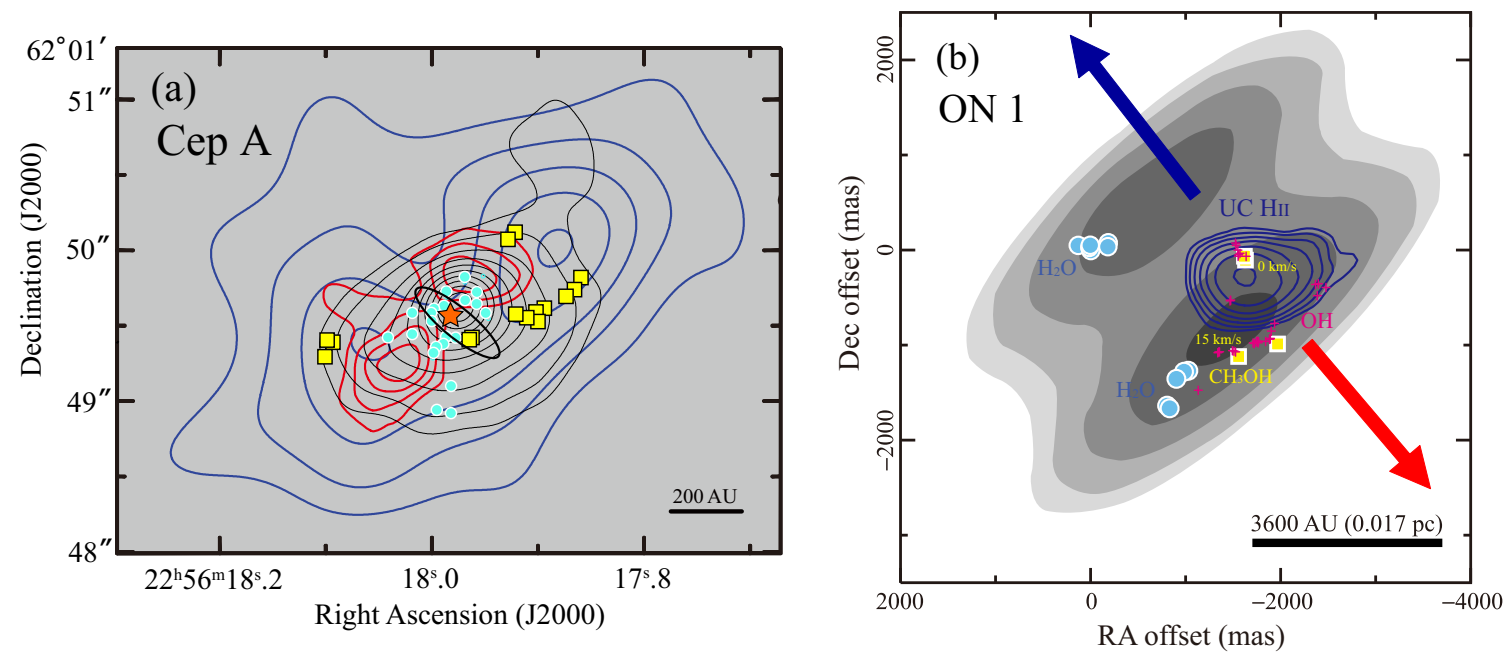

Figure 1: (a) The methanol maser spots for Cep A (square [10]) superposed on water maser (circle: with VLA [11), $335 \mathrm{GHz}$ continuum (black contours [12), $\mathrm{NH}_{3}(4,4)$ emission (blue contours [12]), and $\mathrm{SO}_{2}$ emission (red contours [5]). A thick inclined ellipse shows a radio jet at $22 \mathrm{GHz}$ ([2], 11]), and a star-symbol indicates the peak of $43 \mathrm{GHz}$ continuum emission [2]. (b) The methanol maser spots for ON 1 (square) superposed on water maser (circle [7), hydroxyl maser (cross [3), 8.4 GHz continuum (blue contours 10), and $345 \mathrm{GHz}$ continuum (gray scale 9). Arrows indicate the direction of $\mathrm{H}^{13} \mathrm{CO}^{+}$and $\mathrm{SiO}$ bipolar outflow $[6$ and colors correspond to red- and blue-shifted features. The numbers show approximately radial velocities of each methanol maser spot.

\section{References}

[1] A. L. Argon, M. J. Reid, \& K. M. Menten 2000, ApJSS, 129, 159

[2] S. Curiel, et al. 2006, ApJ, 638, 878

[3] V. L. Fish, \& M. J. Reid 2007, ApJ, 670, 1159

[4] J. A. Green, A. M. S. Richards, W. H. T. Vlemmings, P. Diamond, \& R. J. Cohen 2007, MNRAS, 382, 770

[5] I. Jiménez-Serra, J. Martín-Pintado, A. Rodríguez-Franco, C. Chandler, C. Comito, \& P. Schilke 2007, ApJ, 661, L187

[6] M. S. N. Kumar, M. Tafalla, \& R. Bachiller 2004, A\&A, 426, 195

[7] T. Nagayama, A. Nakagawa, H. Imai, T. Omodaka, \& Y. Sofue 2008, PASJ, 60, 183

[8] N. A. Patel, et al. 2005, Nature, 437, 109

[9] Y.-N. Su, et al. 2004, ApJ, 616, L39

[10] K. Sugiyama, et al. 2008, PASJ, 60, 1001

[11] J. M. Torrelles, J. F. Gómez, L. F. Rodríguez, S. Curiel, P. T. P. Ho, \& G. Garay 1996, ApJ, 457, L107

[12] J. M. Torrelles, N. A. Patel, S. Curiel, P. T. P. Ho, G. Garay, \& L. F. Rodríguez 2007, ApJ, 666, L37.

[13] K. J. E. Torstensson, H. J. van Langevelde, \& S. Bourke 2007, Proceedings of IAU Symp., 242, 178 